\title{
LACUNAS E TENDÊNCIAS DA PESQUISA GLOBAL SOBRE LIXO ZERO NA GESTÃO DE RESÍDUOS
}

\author{
Rafaela da Silva Limons da Cunha ${ }^{1}$, Carlos Mello Garcias ${ }^{2}$ \\ ${ }^{1}$ Professora dos cursos de Engenharia Ambiental e Engenharia Civil; Doutoranda do \\ Programa de Pós-Graduação em Gestão Urbana da PUCPR, Curitiba-PR, Brasil \\ e-mail: rafaela.limons@pucpr.br \\ ${ }^{2}$ Professor Doutor do Programa de Pós-Graduação em Gestão Urbana da PUCPR, Curitiba- \\ PR, Brasil
}
Recebido em: 04/10/2019 - Aprovado em: 30/11/2019 - Publicado em: 15/12/2019
DOI: 10.18677/EnciBio_2019B36

\begin{abstract}
RESUMO
Este estudo teve como objetivo investigar o perfil da pesquisa global sobre lixo zero na gestão de resíduos como forma de selecionar artigos relevantes que venham subsidiar futuras pesquisas. $\mathrm{O}$ trabalho mescla uma análise descritiva dos dados coletados nos artigos com uma análise bibliométrica, o que permitiu identificar alguns dos mais ativos artigos, periódicos, países, instituições e autores na área, além das redes de colaboração. A busca de artigos na base de dados Scopus possibilitou a identificação de um portfólio com 140 publicações acerca do tema e, concluiu-se que o campo de conhecimento relacionado ao lixo zero têm crescido de forma expressiva nos últimos anos. Na análise de coautoria observou-se fragmentação e descentralização. Conforme resultados dos clusters, na ocorrência de palavras-chave foram determinados cinco grupos: economia circular, resíduos municipais, lixo zero, gestão e disposição de resíduos. Os resultados deste estudo evidenciam o lixo zero como um conceito desafiador que ainda requer desenvolvimento para afirmar sua definição, limites, e práticas associadas. Foram identificadas possíveis lacunas nesta área de pesquisa: lixo zero com foco nos resíduos de alimentos, políticas públicas, métodos para medição da prevenção de resíduos e a escassez de envolvimento de atores-chave na gestão.
\end{abstract}

PALAVRAS-CHAVE: gestão de resíduos, lixo zero, redes de colaboração

\section{LACQUINS AND TRENDS OF GLOBAL RESEARCH ON ZERO WASTE IN WASTE MANAGEMENT}

\begin{abstract}
This study has investigated the profile of the global research on Zero Waste in waste management as a way to select relevant articles that may provide the grounds for future research. This paper mixes a descriptive analysis of the data collected in the articles and a bibliometric analysis, which allowed for the identification of some of the most active articles, journals, countries, institutions and authors in the area, as well as collaboration networks. The search for articles in the Scopus database enabled the identification of a portfolio with 140 publications on the subject and, one was able to conclude that the field of knowledge related to Zero Waste has grown significantly in recent years. In the coauthorship analysis, fragmentation and decentralization. According to cluster results, five main groups have been
\end{abstract}


determined regarding word occurrence: circular economy, municipal waste, zero waste, management and waste disposal. The results of this study evidence zero waste as a challenging concept that still requires development to assert its definition, boundaries, and associated practices. Possible gaps in this area of research were identified, zero waste focus on food waste, public policies, methods to measure waste prevention and the lack of involvement of key in management.

KEYWORDS: waste management, zero waste, collaboration networks

\section{INTRODUÇÃO}

Entre os diferentes impactos ambientais associados ao meio urbano, pode-se ressaltar a gestão de resíduos sólidos como o que há de mais desafiador para se atingir a sustentabilidade ambiental nas cidades, já que, se não forem gerenciados adequadamente, provocarão impacto negativo na qualidade de vida da população, além de degradação do solo, da água e do ar e, consequentemente, da saúde humana (SCARLAT et al., 2015).

Os resíduos sólidos tornaram-se uma das questões ambientais globais, e o desenvolvimento de soluções inteligentes e eficazes tem constituído expressivo desafio para os gestores públicos e a própria sociedade pela busca do desenvolvimento sustentável (URBAN, 2016). As dificuldades com os resíduos sólidos nas cidades localizadas nos países em desenvolvimento são influenciadas pelo alto custo de gestão, pela crescente geração e pelo desafio de fazer com que este tema seja tratado como parte do planejamento urbano de uma localidade (LEAL FILHO et al., 2016).

A crescente produção de resíduos é um dos impactos provocados pela rápida urbanização, desenvolvimento econômico e tecnológico que têm afetado o estilo de vida e consumo (ZAMAN, 2015; WEETMAN, 2019). A geração mundial de resíduos duplicou no período de 2000 até 2010 , passando de 0,68 bilhão tonelada/ano para 1,3 bilhão tonelada/ano. A estimativa é de que no ano de 2050 a geração será de 4,2 bilhões de tonelada ano (HOORNWEG; BHADA-TATA, 2012).

A necessidade de reduzir a geração de resíduos fez com que nos últimos anos, o conceito de lixo zero tenha ganhado a atenção de instituições, estudiosos e empresas. Segundo Curran e Williams (2012), o lixo zero refere-se a um conceito unificador que abraça uma série de medidas que visam eliminar os resíduos e incitar os pensamentos tradicionais de geração e disposição. Este conceito tem como intenção estabelecer uma abordagem preventiva e circular dos materiais, mostrando que o caminho a ser seguido na gestão de resíduos deve ter como foco a prevenção na geração, e não como é feito atualmente, somente com o foco no tratamento e disposição final destes resíduos produzidos (SONG et al., 2015; ZAMAN; SWAPAN, 2016; VELEVA et al., 2017).

A análise bibliométrica é uma técnica quantitativa que permite promover o conhecimento do campo analisado, fornecendo aos pesquisadores uma base sólida para posicionar contribuições atuais e detectar novas linhas para pesquisas futuras (ARAÚJO, 2006; MUELLER, 2013).

Neste sentido, o objetivo geral desta pesquisa é apresentar um mapeamento bibliométrico dos artigos publicados na base de dados Scopus sobre lixo zero, de forma que seja possível identificar o nível em que se encontram atualmente as pesquisas científicas acerca do tema. Será apresentado também os temas discutidos pelos autores mais citados, com o intuito de identificar tendências e lacunas de pesquisa. 


\section{MATERIAL E MÉTODOS}

No que tange à abordagem do problema, esta pesquisa pode ser classificada como mista: qualitativa e quantitativa. Esta pesquisa mescla uma análise descritiva dos dados coletados nos artigos com o objetivo de analisar e quantificar o comportamento e o desenvolvimento dos trabalhos no rol das publicações e uma análise bibliométrica, que foi realizada com o objetivo de identificar as redes de colaboração.

\section{Etapas da pesquisa}

A base de dados escolhida foi a Scopus, por ser considerado o principal banco de dados bibliográficos do mundo. Sendo uma das maiores bases existentes, contém resumos e citações e suas publicações revisadas por pares, o que facilita a implementação de dados bibliométricos confiáveis (MORIOKA; CARVALHO, 2016; NOBRE; TAVARES, 2017).

Definiu-se uma sequência de fases para $o$ andamento da análise descritiva e bibliométrica deste trabalho. A primeira etapa se refere à consulta propriamente dita, com a definição das palavras-chave para a análise. Para identificar estudos que versam sobre Lixo Zero, o processo de busca foi executado utilizando-se as palavras-chave "zero waste" (Lixo Zero), "municipal solid waste" (resíduo sólido municipal), "waste management" (gestão de resíduos), "indicators" (indicadores) e o operador boleano "AND" aplicado para "título do artigo, resumo e palavra-chave".

O Quadro 1 apresenta a quantificação dos resultados para cada seleção de palavras-chave.

QUADRO 1 - Resultados da pesquisa bibliométrica

\begin{tabular}{|c|c|c|c|c|}
\hline Zero Waste & $\begin{array}{c}\text { Municipal } \\
\text { Solid Waste }\end{array}$ & $\begin{array}{c}\text { Waste } \\
\text { Management }\end{array}$ & Indicators & Scopus \\
\hline$x$ & $x$ & & & 33 \\
\hline$x$ & & $x$ & & 134 \\
\hline$x$ & & & $\mathrm{x}$ & 14 \\
\hline $\mathbf{x}$ & $x$ & $x$ & & 30 \\
\hline $\mathbf{x}$ & $x$ & & $x$ & 2 \\
\hline$x$ & & $x$ & $x$ & 11 \\
\hline $\mathbf{x}$ & $x$ & $x$ & $x$ & 2 \\
\hline
\end{tabular}

Fonte: os autores (2019).

$\mathrm{Na}$ segunda etapa, foram definidos e aplicados os filtros para delimitação da amostra de publicações a ser estudada. No que diz respeito à tipologia de resultado, indicou-se a modalidade "artigo". Não se aplicou restrição cronológica e de idiomas. O objetivo não era realizar restrições na área temática, mas, após testes previamente realizados na base, resolveu-se excluir quatro áreas, pois fugiam fortemente do escopo desta pesquisa, sendo: 1. Chemistry; 2. Medicine; 3. Biochemistry, Genetics and Molecular Biology e 4. Pharmacology, Toxicology and Pharmaceutics.

Após a busca na base de dados, os 226 artigos encontrados foram importados para o software Mendeley para eliminação de artigos duplicados. O resultado foi uma amostra final de 140 artigos que foram analisados nesta pesquisa. 
$\mathrm{Na}$ terceira etapa, os aspectos foram analisados quantitativamente objetivando a contextualização da produção científica e finalmente, na quarta etapa foi realizada a análise qualitativa, que foi desenvolvida com a construção de diagramas bibliométricos para visualização de coautorias, citações e cocitações para se identificar as redes de colaboração. Também foi identificada a co-ocorrência de termos e de palavras-chave. Para o tratamento destes dados, foi utilizado o software bibliométrico VOSviewer.

\section{RESULTADOS E DISCUSSÃO}

Nesta etapa são apresentados os resultados da análise descritiva realizada nesta pesquisa com a apresentação da distribuição e evolução de artigos ao longo do tempo, principais periódicos e autores, principais temas e identificação dos países e instituições mais atuantes neste campo de estudo.

\section{Distribuição e evolução de artigos ao longo do tempo e periódicos}

A Figura 1 ilustra o período em que os 140 artigos foram publicados entre os anos de 1975 e 2018 com o tema central Lixo Zero. Observa-se um crescimento no número de publicações a partir do ano de 2006, destacando-se como períodos mais expressivos em termos de publicação os anos de 2011, 2013, 2016, 2017 e 2018. Ainda é possível observar que $37 \%$ dos artigos foram publicados nos últimos três anos, ilustrando uma tendência de crescente interesse pelo tema.

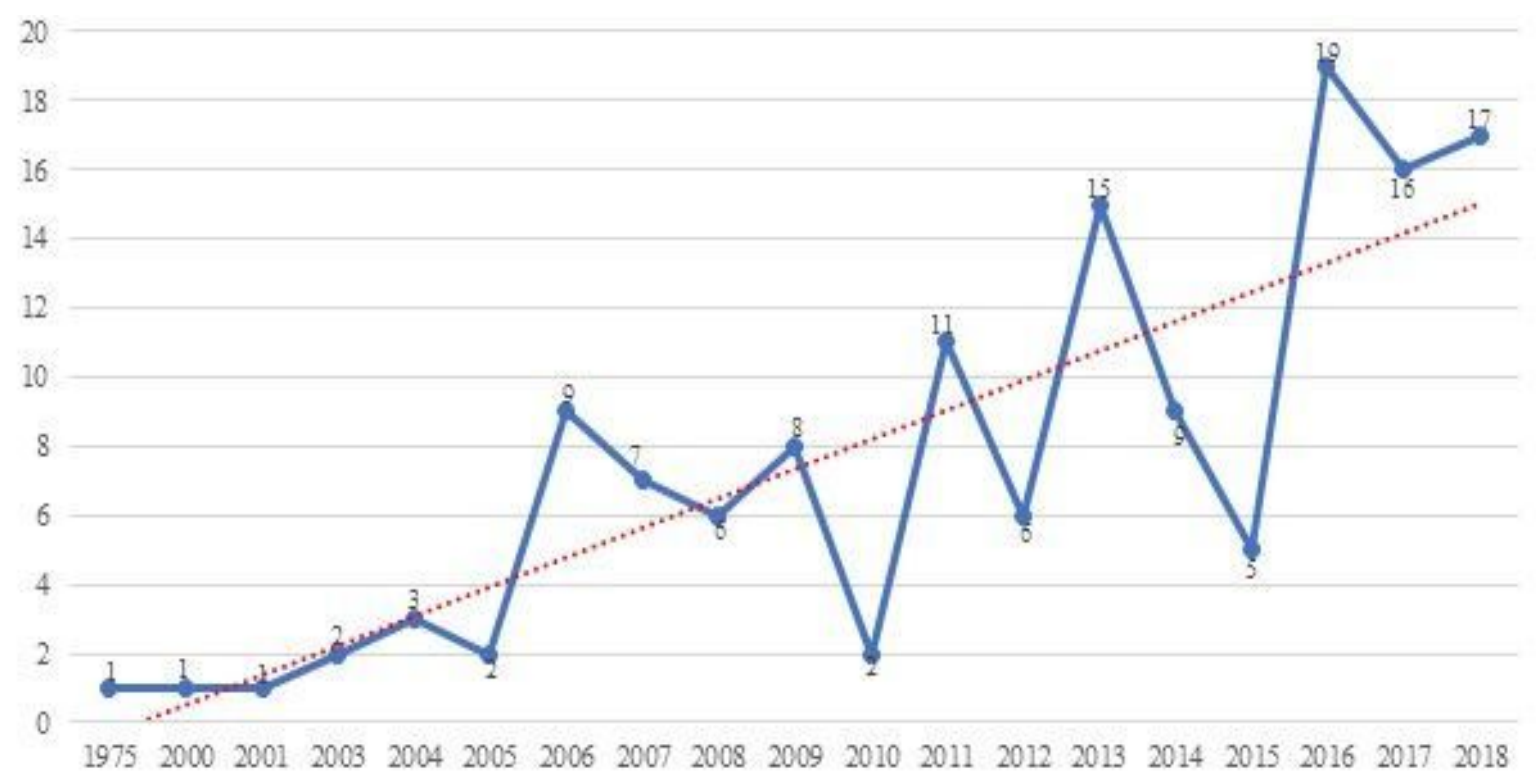

FIGURA 1 - Evolução do número de publicações ao longo dos anos. Fonte: os autores (2019).

No presente estudo foi constatado que o primeiro artigo publicado na área analisada foi em 1975. Foi escrito por Lawrence K. Wang e John Y. Yang, e teve como objetivo geral propor um processo de reciclagem com um sistema de custo efetivo para alcançar a descarga de resíduos "zero" de uma planta de purificação de água.

Os 140 artigos identificados foram publicados em 82 revistas diferentes. $\mathrm{Na}$ Figura 2 estão apresentados os periódicos que publicaram no mínimo dois artigos sobre Lixo Zero: são 12 revistas científicas. Aqueles periódicos que obtiveram no 
mínimo duas publicações no período formam $50 \%$ da amostra total, indicando que os trabalhos sobre o tema estão sendo publicados em diferentes revistas e áreas de interesse.

No entanto, se for considerado somente os periódicos com mais de cinco publicações, observa-se o fenômeno descrito pela Lei de Bradford: $40 \%$ das publicações, ou 56 artigos, acerca do tema se encontram em somente seis periódicos, enquanto outros 76 periódicos contêm $60 \%$ das publicações, com 84 artigos. O que se verifica é um núcleo de difusão de conhecimentos sobre a temática do Lixo Zero. A revista Waste Management foi identificada como o periódico de maior contribuição para as pesquisas relacionadas ao tema, com 12,9\% das publicações.

Machado Junior et al. (2016) retratam a utilidade da Lei de Bradford no processo de aquisição e descarte de periódicos. Os autores evidenciam que o processo de investigação desenvolvido por pesquisadores também se beneficia deste princípio, pois, pesquisando-se em uma pequena base de revistas especializadas sobre um tema, é possível identificar uma quantidade significativa de artigos sobre o assunto de interesse. Fora deste núcleo de revistas especializadas, o pesquisador deverá consultar um volume muito maior de revistas para encontrar a mesma quantidade de artigos deste assunto.

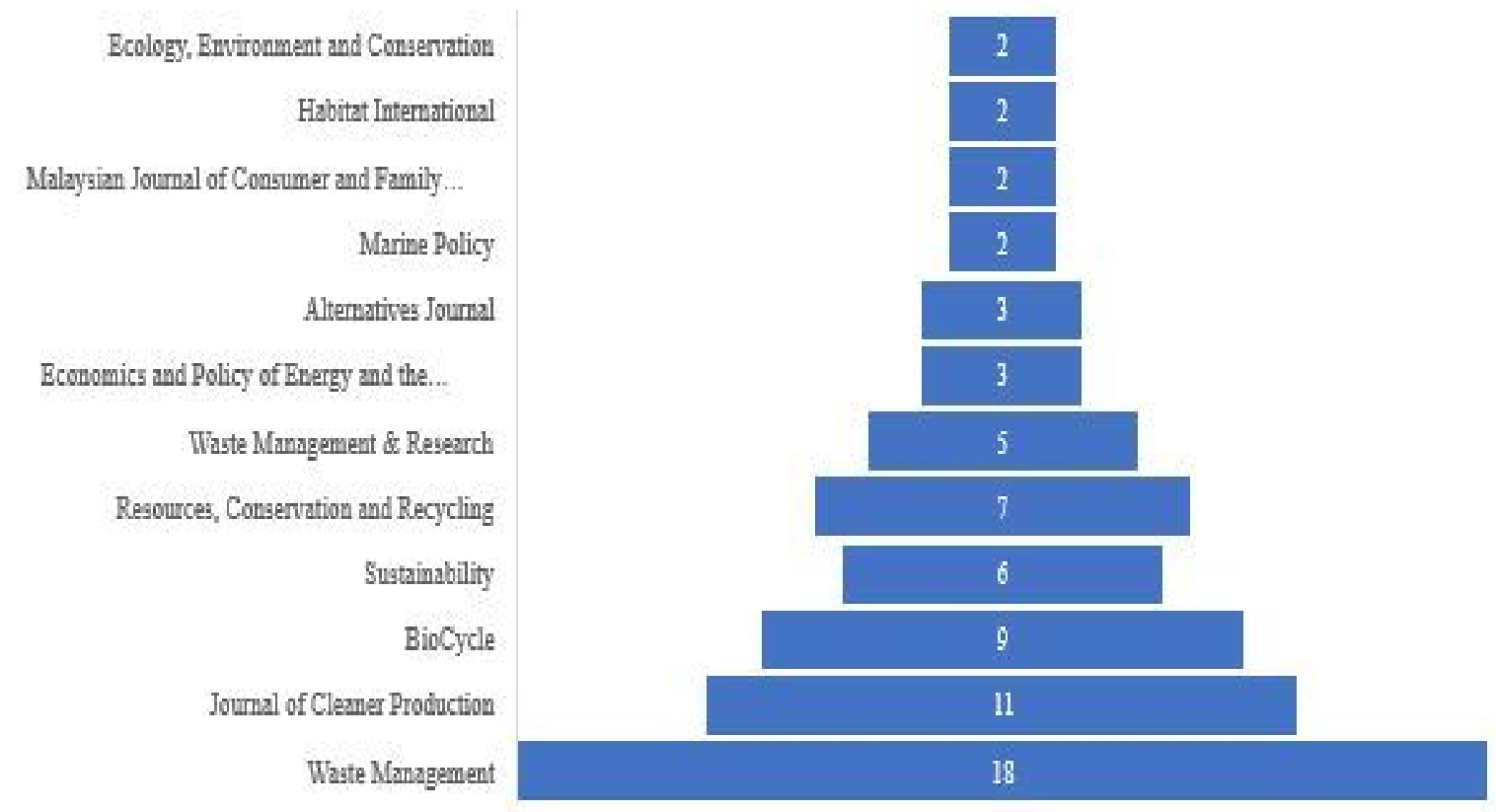

FIGURA 2 - Número de artigos publicado por periódico. Fonte: os autores (2019).

Ao realizar o levantamento dos principais periódicos que publicam sobre determinado tema, tais informações servem como base para pesquisas futuras. Esta análise servirá para que autores interessados em desenvolver pesquisas sobre um determinado tema possuam uma base para encontrar os dados necessários e, de acordo com a Lei de Bradford, pode-se definir estas 12 como "revistas principais" na polarização do tema Lixo Zero.

\section{Autores}

Quanto à análise de autoria, a pesquisa identificou 384 autores relacionados 
às produções analisadas. Para a elaboração da Tabela 1, foram considerados os autores que publicaram no mínimo três artigos. Sendo assim, foram listados os quatro autores mais prolíficos na produção de artigos acerca do tema proposto nesta pesquisa. Os autores apresentados na Tabela 1 assinam 21 das 140 publicações analisadas, representado $15,1 \%$ da amostra.

TABELA 1 - Produtividade dos autores analisados

\begin{tabular}{ccc}
\hline Autor & $\begin{array}{c}\text { Número de } \\
\text { artigos }\end{array}$ & $\%$ \\
\hline Zaman & 9 & 6,4 \\
Lehmann & 6 & 4,3 \\
Basri & 3 & 2,2 \\
Williams & 3 & 2,2 \\
\hline Total & 21 & 15,1 \\
\hline
\end{tabular}

Fonte: os autores (2019).

O autor Zaman, pesquisador do Instituto de Política de Sustentabilidade da Curtin University, Austrália, foi identificado como o maior contribuinte para as pesquisas acerca do tema abordado nesta bibliometria. $O$ pesquisador é o autor principal de sete artigos, sendo o secundário em outros dois artigos em conjunto com o pesquisador Lehmann. Dos seis artigos associados ao pesquisador Steffen Lehman, em três ele é o autor principal e nos outros três ele é secundário ao pesquisador Atiq Uz Zaman. Estes dois pesquisadores podem ser considerados como os mais prolíficos nesta temática, sendo que o foco da maioria de suas pesquisas é o resíduo urbano.

Os pesquisadores Noor Ezlin Ahmad Basri e lan D Williams se apresentam como coautores de três pesquisas cada um. São afiliados à Universidade Kebangsaan (Malásia) e à Universidade de Southampton (Reino Unido), respectivamente.

Os resultados desta análise indicam que existem alguns pesquisadores trabalhando com núcleos de referência sobre Lixo Zero, constatando-se, assim, o uso da Lei Bibliométrica de Lotka, uma vez que, dos 384 autores identificados nas 140 publicações com o foco em Lixo Zero, apenas $1,04 \%$ escreveu $15,1 \%$ dos artigos, enquanto os outros 380 autores têm participação em somente um ou dois artigos, representando o restante das $84,9 \%$ das publicações, ou seja, há grande concentração da produção científica em alguns poucos autores.

\section{Países e instituições de pesquisa}

Em relação à distribuição geográfica dos documentos publicados, observouse, conforme mostra a Figura 3, que o desperdício zero é tema de pesquisa em diferentes países. Dos 140 artigos publicados, tem-se 42 países envolvidos, considerados autor principal e coautores de cada artigo selecionado. Os Estados Unidos apresentaram 23 artigos, seguido da China e Austrália, com 13 artigos, Reino Unido (12), Malásia (11), Índia (9), Grécia (7), Canadá (5), e África do Sul e Japão (4). 


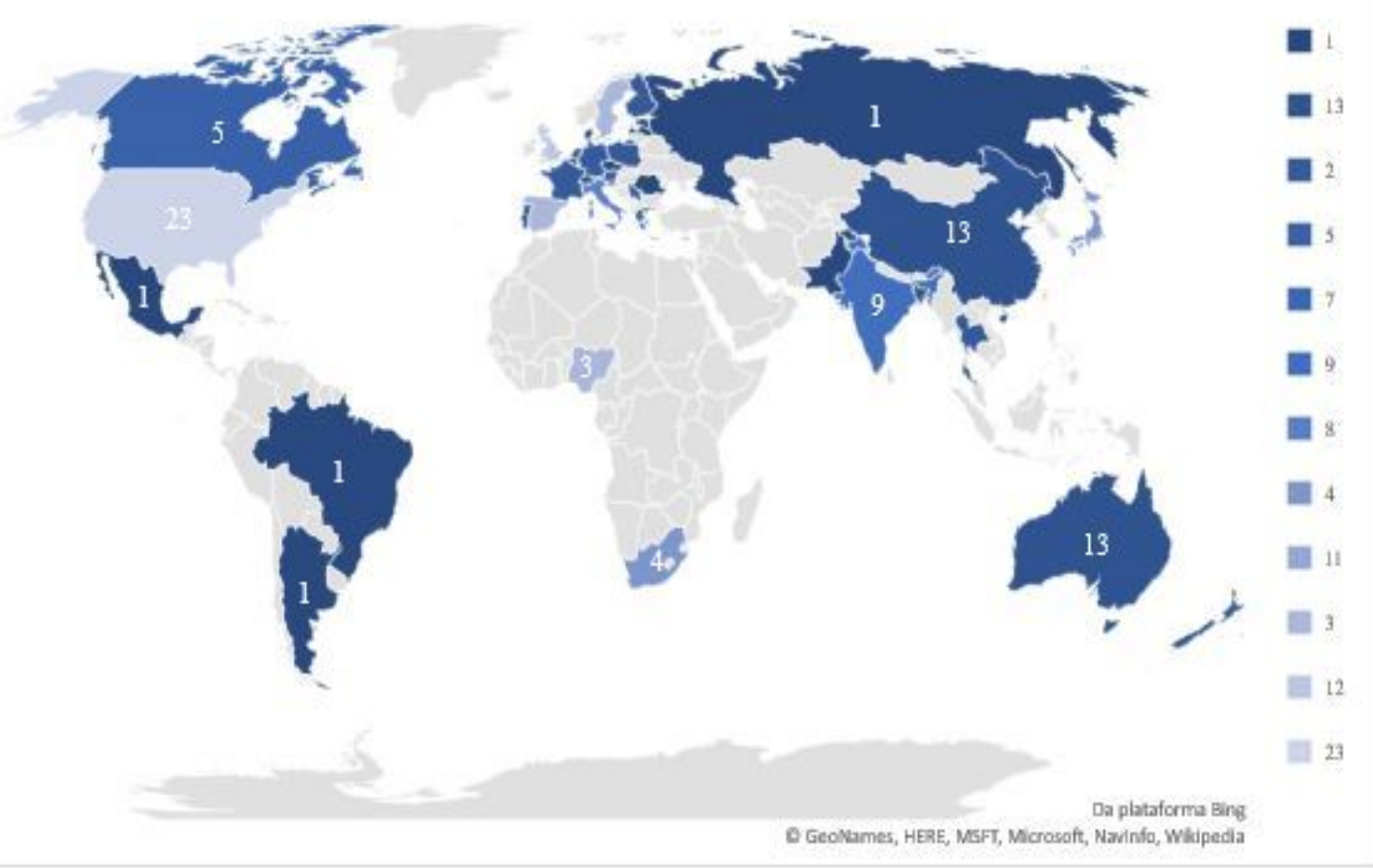

FIGURA 3 - Quantidade de publicações por país. Fonte: os autores (2019).

Com base nesta figura, é possível observar uma difusão dos estudos de Lixo Zero em diferentes partes do planeta, com maior presença na Ásia. Observa-se uma presença baixa de pesquisas na América do Sul e na África, o que seria fundamental para entender as particularidades de cada localidade, visto que é gestão de resíduos sólidos depende consideravelmente das condições locais.

É importante ressaltar que, dos 23 artigos com autores de afiliação americana, nove deles foram publicados na revista Biocycle, que não publica desde 2011. No entanto, mesmo desconsiderando estes artigos, os Estados Unidos estão em primeiro lugar na quantidade de publicações, com 14 artigos sobre a temática analisada.

Entre as instituições mais prolíferas, é possível apontar aquelas que estão localizadas nos países mais produtivos, como Austrália, Malásia e Estados Unidos. Todavia, observa-se a participação de diferentes instituições em cada publicação, visto que foram identificadas 174 instituições diferentes no conjunto de 42 países com pesquisas, além daqueles artigos sem identificação de afiliação. Os resultados da análise identificaram a Universiti Kebangsaam, a University of South Australia, seguida da Aristotle Universit of Thessabniki como as instituições mais produtivas em autoria ou coautoria, estando presentes em 17, 15 e 13 publicações, respectivamente.

\section{Redes de Colaboração}

Para detectar e interpretar padrões e vínculos entre pesquisadores e regiões geográficas que estão produzindo sobre a temática Lixo Zero, foi realizado nesta etapa um conjunto de análises de redes.

Para as consultas de rede de colaboração, utilizou-se o VOSviewer como apoio para o tratamento dos dados. O VOSviewer é um software gratuito de análise bibliométrica que apresenta a conexão entre termos, autores, e outros apectos relacionados, proporcionando a divisão em grupos denominados clusters (VAN ECK; WALTMAN, 2010; FAHIMNIA et al., 2015). 


\section{Análises de coautoria}

Com a análise de redes de coautoria, é possível identificar a relação entre pesquisadores, institutos ou países nas suas publicações (VAN ECK; WALMAN, 2010; VAN ECK; WALMAN, 2017). Foi construída uma rede de relacionamento considerando os 140 artigos da amostra. Cada autor é representado na rede por um nó, de acordo com o número de artigos de sua autoria, e suas relações de coautoria com os demais autores são representadas pelas arestas, conforme mostra a Figura 4.

Para a elaboração desta rede com apoio do VOSviewer, foram considerados os autores com no mínimo dois documentos publicados. Dos 384 autores, tal restrição produziu uma rede com 17 autores, distribuídos em nove clusters de colaboração em pesquisa, com destaque para grupos contendo poucos pesquisadores interligados.

Analisando a figura, é possível identificar fragmentação e descentralização, sendo difícil afirmar que exista uma rede de colaboração a ser considerada nestes trabalhos. Foi possível identificar quatro clusters com a colaboração entre autores. No cluster vermelho e verde, observam-se periódicos com participação de autores da China, enquanto no cluster azul e amarelo, são pesquisadores da Austrália e Reino Unido, respectivamente.

O tamanho dos círculos indica a quantidade de artigos de cada autor na amostra, com destaque para os autores Zaman e Lehmann. O cluster com mais nós e, portanto, com mais autores colaborando entre si, aparecem na cor vermelha, constituído por quatro autores. Observam-se cinco clusters com somente um autor.

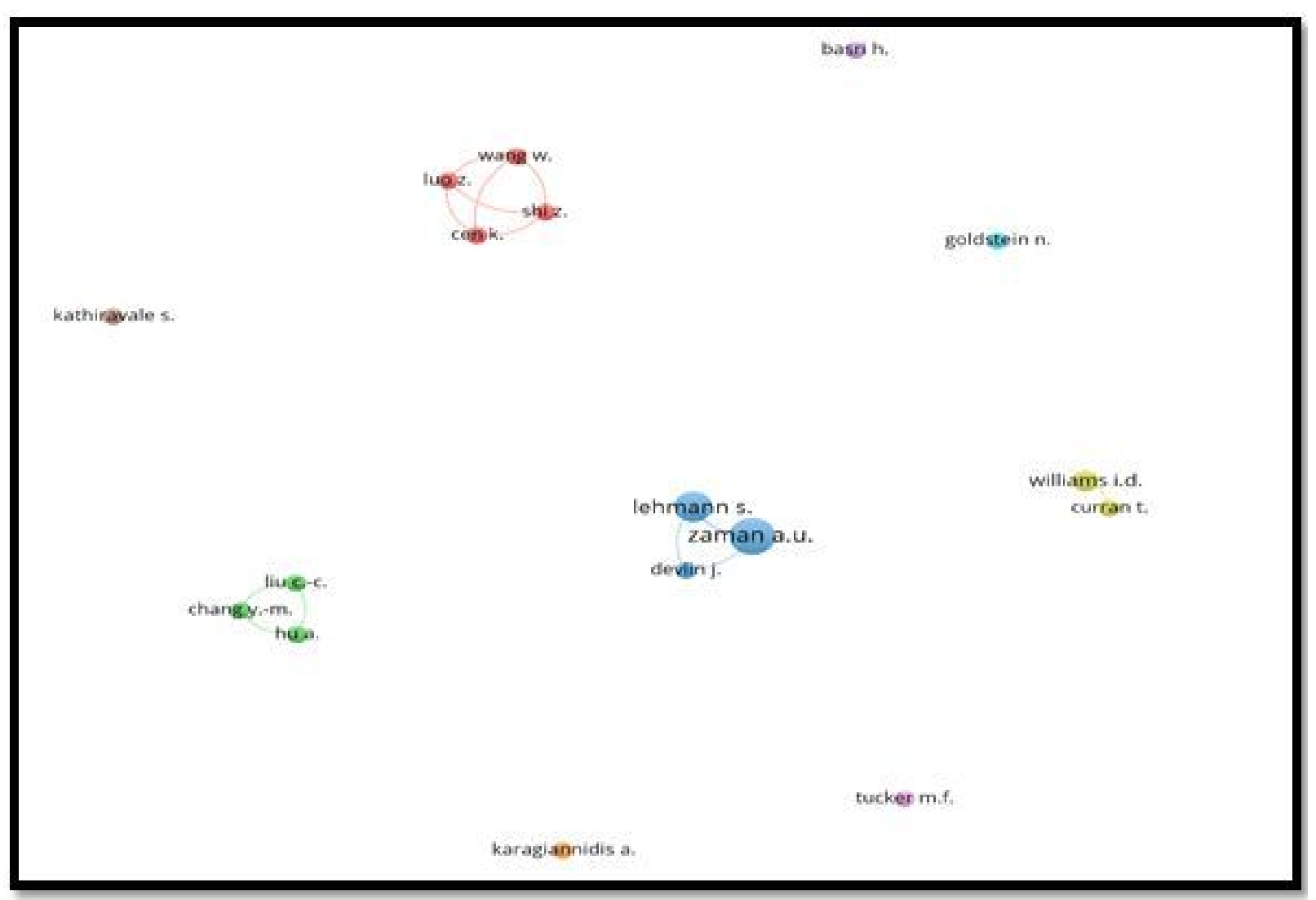

FIGURA 4 - Redes de coautoria. Fonte: os autores (2019). 
Os autores presentes no cluster azul e vermelho pesquisam fundamentalmente a temática Lixo Zero no âmbito dos resíduos sólidos urbanos, enquanto os pesquisadores dos clusters vermelho e amarelo se aprofundaram no domínio na gestão de resíduos industriais.

Visando identificar os países cujos autores mais publicaram em colaboração, a Figura 5 mostra a principal rede de países mapeados. Foram considerados somente países com no mínimo duas publicações em conjunto, neste caso 12. Com base na figura, observa-se a formação de quatro clusters de colaboração nas coautorias dos artigos: o vermelho, liderado pelo Reino Unido, tem o maior número de publicações e inclui Áustria, Polônia e Portugal; o amarelo, é composto por Austrália e França; o azul, por China, Itália e Suécia e, para finalizar, o cluster verde é composto por Ciprus, Itália e Estados Unidos.

É possível verificar na figura, pelo tamanho do círculo, os países com o maior número de artigos publicados; no entanto, o que importa neste caso é a colaboração: o Reino Unido foi o país mais afiliado, vinculado a seis outros países. Atrás deste estão Grécia (quatro ligações) e Polônia (três ligações).

Estes resultados indicam que as vantagens de uma colaboração internacional não se limitam à ampliação da rede, troca e compartilhamento de conhecimento; esta também forma uma estratégia eficaz de classificação. Por exemplo, países como Malásia, Índia, África do Sul, Austrália e Canadá estão entre os países que mais publicaram sobre o tema; no entanto, apresentam baixa colaboração internacional. Reforçando o aspecto da colaboração internacional, os três primeiros países citados apresentam todas as publicações somente com autores do seu país, sugerindo uma forte colaboração intra-país.

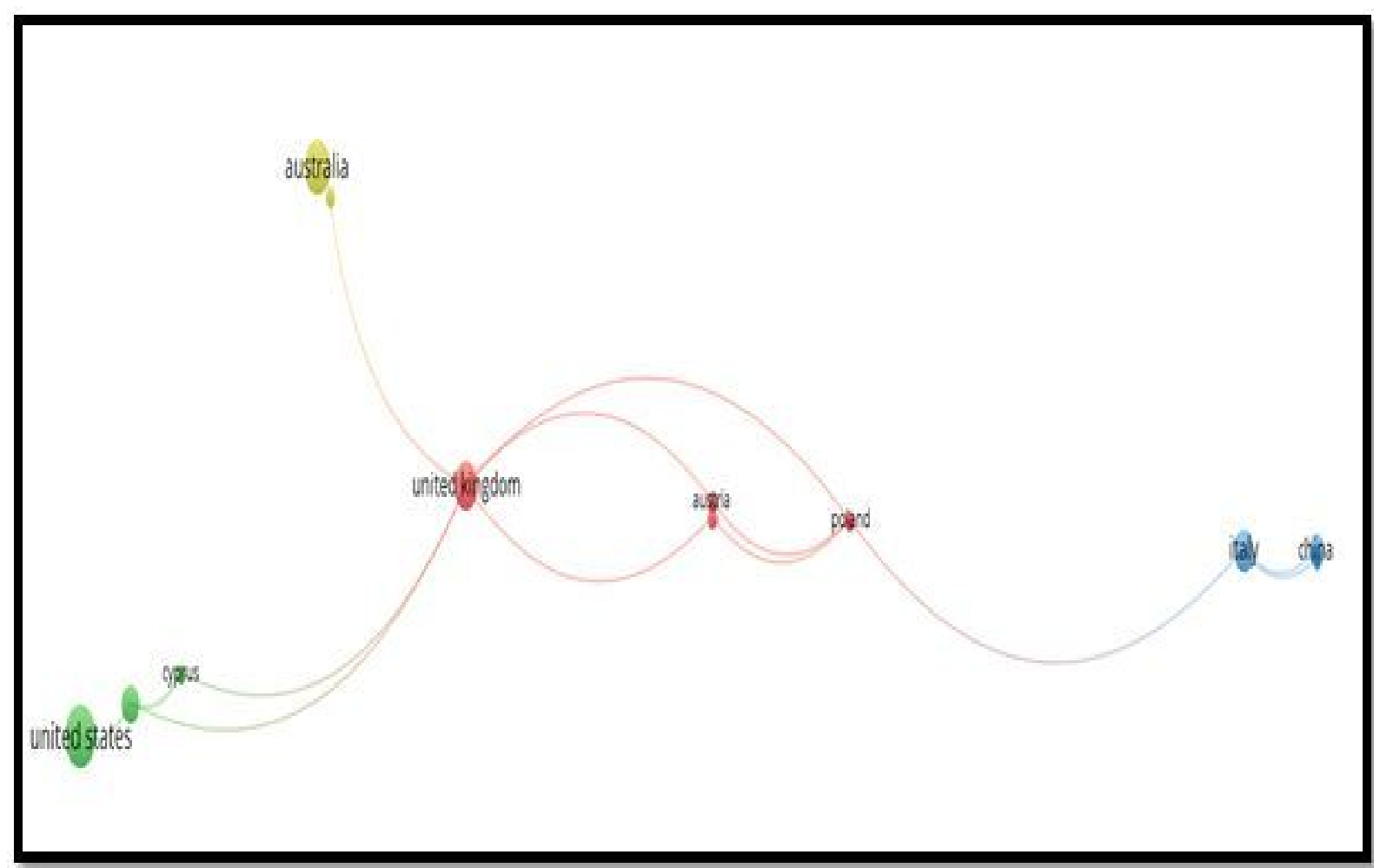

FIGURA 5 - Diagrama de relacionamento entre países. Fonte: os autores (2019). 


\section{Rede de cocitação}

A análise de cocitações é determinada pelo número de vezes que dois artigos são citados em conjunto. Assim sendo, a frequência de cocitação estabelece uma rede de similaridade entre dois autores ou documentos, deixando evidente uma estrutura de conhecimento percebida pelo citante (VAN ECK; WALTMAN, 2017).

Obteve-se que, da amostra analisada, 7280 autores foram citados. Para visualização da rede, foram considerados somente autores que tivessem sido citados no mínimo oito vezes nas referências desses artigos, totalizando, assim, um quantitativo de 40 autores. Na Figura pode-se observar a rede de cocitação sendo possível identificar os principais pesquisadores que contribuíram para o desenvolvimento intelectual sobre o tema, além de suas inter-relações.

É possível observar na figura, a formação de cinco clusters com forte ligação entre diferentes pesquisadores, sendo Lehmann, Zaman, Geng e Fujita talvez os quatro autores mais ativos e influentes em gestão de resíduos com enfoque em Lixo Zero.

Os pesquisadores Zaman e Lehmann, aparecem como os autores com o maior número de citações em cocitações: 34 cada um. No entanto, considerando que nesta análise a ordem de importância não se baseia no número de citações, mas na força de relação entre autores na colaboração científica, pode-se incluir Geng, que apresenta 31 citações e reflete 678 relações, enquanto Fujita é citado 13 vezes e reflete 395 ligações e Zaman e Lehmann apresentam 374 e 334 relações, respectivamente.

Zaman e Lehamn, além de serem os autores que mais publicaram sobre Lixo Zero, também são os dois dos autores que mais foram citados em conjunto na amostra dos 140 artigos analisados conforme observado na Figura 6. Eles se centram em estudos que analisam as possibilidades de transformação da prática tradicional de gestão de resíduos em práticas de Lixo Zero.

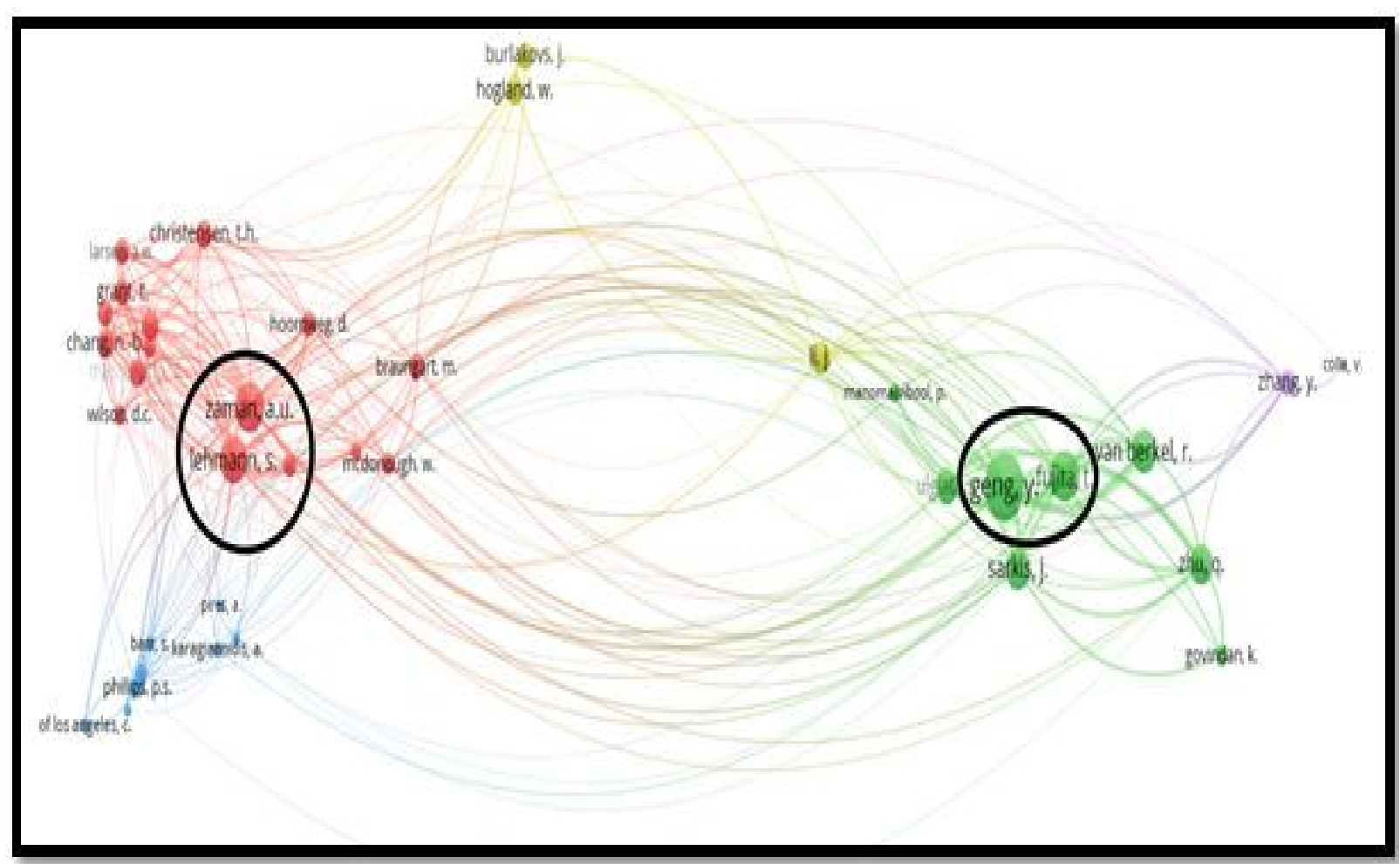

FIGURA 6 - Rede de cocitação. Fonte: os autores (2019). 
$\mathrm{Na}$ busca inicial desta pesquisa não foram encontrados artigos da rede composta por Fujita e Geng, mas eles são comumente citados em conjunto na amostra analisada. O pesquisador Fujita é afiliado à Universidade Toyo, no Japão, enquanto Geng trabalha na Universidade Shangai Jiao Tong, na China. Provavelmente os artigos destes autores não apareceram na busca inicial da base de dados, pois o enfoque de sua pesquisa não é o Lixo Zero. No entanto, os autores publicaram diversos artigos sobre a cidade de baixo carbono e economia circular sustentável, assuntos diretamente ligados ao tema estudado nesta pesquisa.

Concluindo esta análise, é possível identificar o quão influente ou quão impactante é ou foi um pesquisador na comunidade científica da área. Assim, numa pesquisa futura e aprofundada sobre Lixo Zero, é fundamental que estes autores sejam considerados como base para a fundamentação teórica e o desenvolvimento da pesquisa.

\section{Análise de termos e palavras-chave}

Utilizando a função de identificação de termos do VOSviewer, que busca termos tanto no título quanto no resumo dos artigos, foi construído o diagrama de relacionamento, considerando relevantes aqueles que tenham sido citados no mínimo cinco vezes. No total de 1620 termos apresentados, atenderam aos critérios os 54 termos exibidos na Figura 7.

Esta figura mostra quais são os termos de pesquisa mais importantes e como estão interligados. Observa-se que os nós de tamanho maior representam tópicos de tendência e descrevem os conceitos mais recorrentes na literatura. Esta rede é formada por quatro clusters: o azul contém os termos com o maior número de ocorrências e ligações entre eles, como waste management (101), recycling (50), zero waste (40) e municipal solid waste (25). Além disso, observam-se neste cluster termos como food waste, circular economy, e public policy.

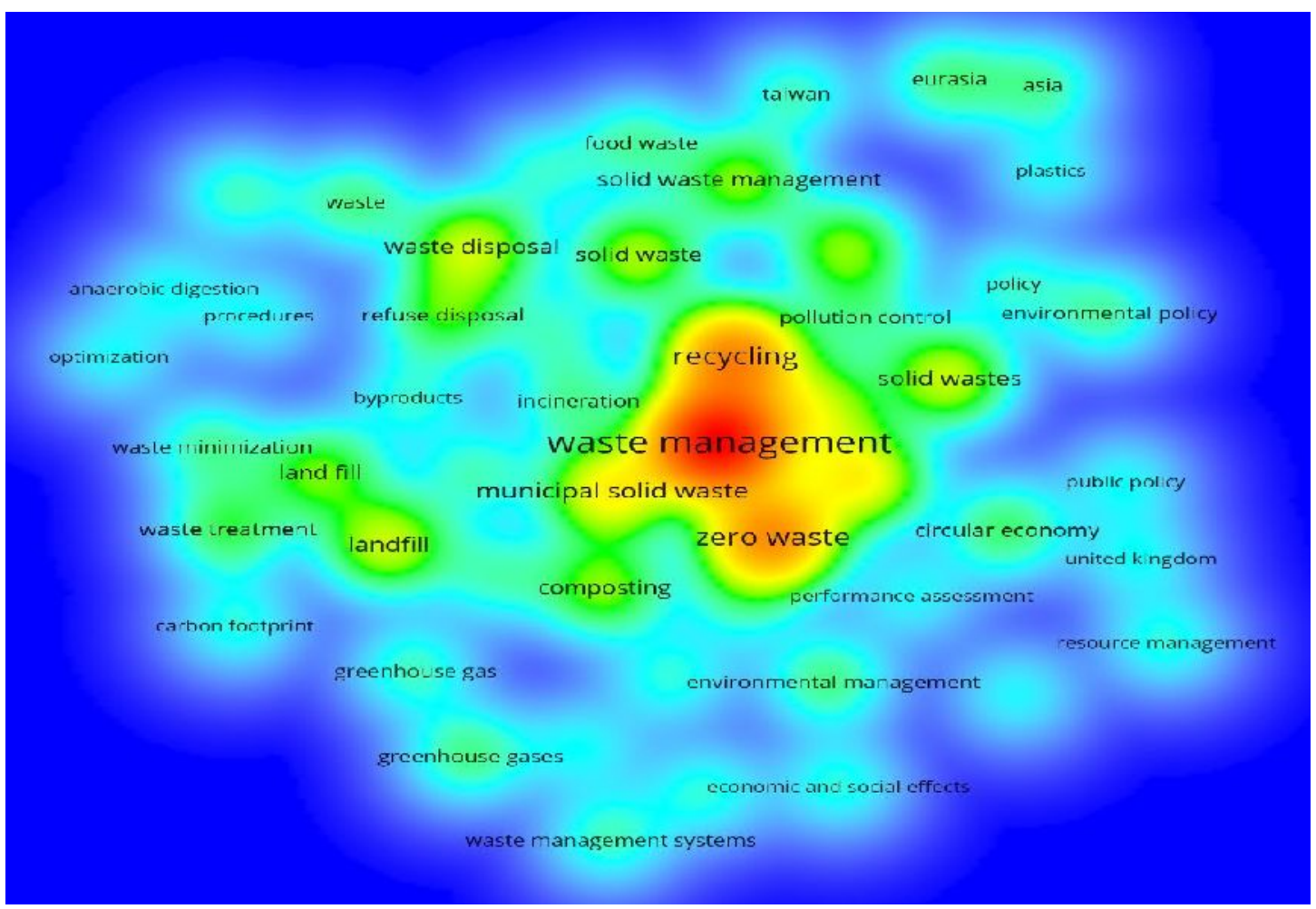

FIGURA 7 - Mapa de densidade de ocorrência dos termos. Fonte: os autores (2019). 
Considerando somente as palavras-chave dos artigos selecionados, as seis palavras-chave com maior ocorrência foram: zero waste (31 ocorrências), waste management (22), recycling (13), sustainability (11), circular economy (10), e municipal solid waste (5). Neste sentido, pode-se identificar possíveis lacunas nesta área de pesquisa, como por exemplo, os termos resíduos de alimentos (food waste), public policy (políticas públicas) e perfomance assessment (avaliação de desempenho) foram discutidos em vários destes artigos, inclusive já identificados em vários como GAP de pesquisa, no entanto, pouco aprofundado como temas principais de pesquisa.

\section{CONCLUSÃO}

Esta análise bibliométrica forneceu um cenário geral das tendências de pesquisa sobre Lixo Zero considerando um universo de 140 documentos que foram analisados a partir do banco de dados Scopus. Foram apontados como temas emergentes a necessidade de métodos para medição da prevenção de resíduos e a escassez de envolvimento de atores-chave na sua gestão. Além disso, foram identificadas possíveis lacunas nesta área de pesquisa, como por exemplo, Lixo Zero com foco nos resíduos de alimentos e a necessidade de políticas públicas nesta área de conhecimento.

Os resultados deste estudo evidenciam o Lixo Zero como um conceito desafiador que ainda requer desenvolvimento para afirmar sua definição, limites, e práticas associadas. Um dos principais desafios identificados nesta revisão é o de estabelecer o Lixo Zero como uma abordagem preventiva, holística e circular dos recursos, e não somente como meta zero de resíduos enviados para aterro e incineração.

Por fim, espera-se que os resultados apresentados neste trabalho permitam novos caminhos de pesquisa a partir do mapeamento realizado. No entanto, é importante considerar que estudos bibliométricos possuem algumas limitações, como, por exemplo: a coleta de dados foi conduzida exclusivamente no banco de dados Scopus e, ao restringir a pesquisa de Lixo Zero aos "títulos, resumos e palavras-chave", o resultado não cobriu todos os estudos relacionados ao tema. Uma possibilidade de pesquisa futura seria a análise combinada de dois ou até mais bancos de dados para comparação dos resultados, e utilizando as novas palavraschave que foram identificadas como possíveis lacunas de pesquisa.

\section{REFERÊNCIAS}

ARAÚJO, C. Bibliometria: evolução histórica e questões atuais. Em Questão, v. 12, n.1, p. 11-32, 2006. Disponível em: $<$ https://seer.ufrgs.br/EmQuestao/article/view/16>.

CURRAN, T.; WILLIAMS, I. A zero waste vision for industrial networks in Europe. Journal of Hazardous Materials, v. 207-208, p. 3-7, 2012. Disponível em: <https://doi.org/10.1016/j.jhazmat.2011.07.122>. doi: 10.1016/j.jhazmat.2011.07.122

FAHIMNIA, B.; SARKIS, J.; DAVARZANI, H. Green supply chain management: A review and bibliometric analysis. International Journal of Production Economics, v. 162, $\quad$ p. $101-114, \quad 2015 . \quad$ Disponível em: <https://doi.org/10.1016/j.ijpe.2015.01.003>. doi: 10.1016/j.ijpe.2015.01.003 
HOORNWEG, D.; BHADA-TATA, P. What a waste: a global review of solid waste management. Urban Development. World Bank, Washington DC, USA, p. 98, 2012. Disponível em: <http://hdl.handle.net/10986/17388>

LEAL FILHO, W.; BRANDLI, L.; MOORA, H.; KRUOPIENE, J.; STENMARCK, A. Benchmarking approaches and methods in the field of urban waste management. Jounal of Cleaner Production, v. 112, Part. 5, n.20, p. 4377-4386, 2016. Disponível em: $\quad$ <https://doi.org/10.1016/j.jclepro.2015.09.065>. doi: 10.1016/j.jclepro.2015.09.065

MACHADO JUNIOR, C.; SOUZA, M.T.S.; PARISOTTO, I.R.S.; PALMISANO, A. As leis da bibliometria em diferentes bases de dados científicos. Revista de Ciências da Administração, v.18, n.44, p.11-123, 2016. Disponível em: <https://doi.org/10.5007/2175-8077.2016v18n44p111>. doi: 10.5007/21758077.2016v18n44p111

MORIOKA, S. N.; CARVALHO, M. M. de. Sustentabilidade e gestão de projetos: um estudo bibliométrico. Production, v. 26, n. 3, p. 656-674, 2016. Disponível em: <http://dx.doi.org/10.1590/0103-6513.058912>. doi: 10.1590/0103-6513.05891

MUELLER, S. Estudos métricos da informação em ciência e tecnologia no Brasil realizados sobre a unidade de análise artigos de periódicos. Liinc em Revista, v.9, n. 1, p. 6-27, 2013. Disponível em: <https://doi.org/10.18617/liinc.v9i1.558>. doi: 10.18617/liinc.v9i1.558

NOBRE, G. C.; TAVARES, B. E. Scientific literature analysis on big data and internet of things applications on circular economy: a bibliometric study. Scientometrics, v. 111, n. 1, p. 463-492, 2017. Disponível em: <https://doi.org/10.1007/s11192-0172281-6>. doi: 10.18617/liinc.v9i1.558

SCARLAT, N.; MOTOLO, V.; DALLEMAND, J.F.; MONFORTI-FERRARIO, F.; MORFOR, L. Evaluation of energy potential of municipal solid waste from African urban areas. Renewable and Sustainable Energy Rewies, v. 50, p. 1269-1286, 2015. Disponível em: <https://doi.org/10.1016/j.rser.2015.05.067> doi: 10.1016/j.rser.2015.05.067

SONG, Q.; LI, J.; ZENG, X. Minimizing the increasing solid waste through zero waste strategy. Journal of Cleaner Production, v. 104, n.1, p.199-210, 2015. Disponível em: $\quad<$ https://doi.org/10.1016/j.jclepro.2014.08.027>.

doi: 10.1016/j.jclepro.2014.08.027

URBAN, R. C. Índice de adequação do gerenciamento de resíduos sólidos urbanos como ferramenta para o planejamento: aplicação no estado de São Paulo. Engenharia Sanitária e Ambiental, v. 21, n. 2, p. 367-377, 2016. Disponível em: <http://dx.doi.org/10.1590/s1413-41522016140543>. doi: 10.1590/s141341522016140543

VAN ECK, N. J.; WALTMAN, L. Software survey: Vosviewer, a computer program for bib-liometric mapping. Scientometrics, v. 84, n. 523, p. 523-538, 2010. Disponível 
em: <https://doi.org/10.1007/s11192-009-0146-3>. doi: 10.1007/s11192-009-0146-3 VAN ECK, N. J.; WALTMAN, L. Citation-based clustering of publications using CitNetExplorer and VOSviewer. Scientometrics, v. 111, n. 2, p. 1053-1070, 2017. Disponível em: <https://doi.org/10.1007/s11192-017-2300-7>. doi: 10.1007/s11192017-2300-7

VELEVA, V.; BODKIN, G.; TODOROVA, S. The need for better measurement and employee engagement to advance a circular economy: Lessons from Biogen's "Zero Waste" journey". Journal of Cleaner Production, v.154, p. 517- 529, 2017. Disponível em: <https://doi.org/10.1016/j.jclepro.2017.03.177>. doi: 10.1016/j.jclepro.2017.03.177

WEETMAN, C. Economia circular: conceitos e estratégias para fazer negócios de forma mais inteligente, sustentável e lucrativa. Tradução de Afonso Celso da Cunha Serra. 1. ed. São Paulo: Autêntica Business, 2019. 512 p.

ZAMAN, A. U. A comprehensive review of the development of zero waste management: lessons learned and guidelines. Journal of Cleaner Production, $\mathrm{v}$. 91, p. 12-25, 2015. Disponível em: <https://doi.org/10.1016/j.jclepro.2014.12.013>. doi: 10.1016/j.jclepro.2014.12.013

ZAMAN, A.U.; SWAPAN, M.S.H. Performance evaluation and benchmarking of global waste management systems. Resources, Conservation and Recycling, v. 114, p.32-41, 2016. Disponível em: <https://doi.org/10.1016/j.resconrec.2016.06.020>. doi: 10.1016/j.resconrec.2016.06.020 